\title{
Risk Factors and Incidence of Acute Ischemic Stroke: A Comparative Study Between Young Adults and Older Adults
}

Urvish K. Patel ${ }^{1}$, Mihir Dave ${ }^{2}$, Anusha Lekshminarayanan ${ }^{3,4}$, Preeti Malik ${ }^{5,6}$, Matthew DeMasi ${ }^{7}$, Sangeetha Chandramohan ${ }^{5}$, Shreejith Pillai ${ }^{8}$, Raghavendra Tirupathi ${ }^{9}$, Shamik Shah ${ }^{10}$, Vishal B. Jani ${ }^{11}$, Mandip S. Dhamoon ${ }^{12}$

1. Public Health and Neurology, Icahn School of Medicine at Mount Sinai, New York, USA 2. Internal Medicine, University of Nevada Reno, School of Medicine, Reno, USA 3. Internal Medicine, Richmond University Medical Center, Staten Island, USA 4. Rehabilitation Medicine, New York Medical College and Metropolitan Hospital Center, New York, USA 5. Public Health, Icahn School of Medicine at Mount Sinai, New York, USA 6. Neurology, Massachusetts General Hospital, Boston, USA 7. Internal Medicine, Albert Einstein College of Medicine, Bronx, USA 8. Internal Medicine, Henry Ford Health System, Detroit, USA 9. Internal Medicine, Keystone Health, Chambersburg, USA 10. Neurology, Stormont Vail Health, Topeka, USA 11. Neurology, Creighton University School of Medicine, Omaha, USA 12. Neurology, Icahn School of Medicine at Mount Sinai, New York, USA

Corresponding author: Urvish K. Patel, dr.urvish.patel@gmail.com

\section{Abstract}

\section{Introduction}

Approximately 5-10\% of strokes occur in adults of less than 45 years of age. The rising prevalence of stroke risk factors may increase stroke rates in young adults (YA). We aimed to compare risk factors and outcomes of acute ischemic stroke (AIS) among YA.

\section{Methods}

Adult hospitalizations for AIS and concurrent risk factors were found in the Nationwide Inpatient Sample database. Weighted analysis using chi-square and multivariable survey logistic regression was performed to evaluate AIS-related outcomes and risk factors among YA (18-45 years) and older patients.

\section{Results}

A total of 4,224,924 AIS hospitalizations were identified from 2003 to 2014, out of which 198,378 (4.7\%) were YA. Prevalence trend of YA with AIS showed incremental pattern over time (2003: 4.36\% to 2014: 4.7\%; pTrend $<0.0001$ ). In regression analysis, the risk factors associated with AIS in YA were obesity (adjusted odds ratio \{aOR\}: 2.26; $\mathrm{p}<0.0001$ ), drug abuse (aOR: $2.56 ; \mathrm{p}<0.0001$ ), history of smoking (aOR: 1.20 ; $\mathrm{p}<0.0001$ ), infective endocarditis (aOR: $2.08 ; \mathrm{p}<0.0001$ ), cardiomyopathy (aOR: $2.11 ; \mathrm{p}<0.0001$ ), rheumatic fever (aOR: 4.27 ; $\mathrm{p}=0.0014$ ), atrial septal disease (aOR: 2.46 ; $\mathrm{p}<0.0001$ ), ventricular septal disease (aOR: 4.99; $\mathrm{p}<0.0001$ ), HIV infection (aOR: 4.36; $\mathrm{p}<0.0001$ ), brain tumors (aOR: 7.89; $\mathrm{p}<0.0001$ ), epilepsy (aOR: 1.43 ; $\mathrm{p}<0.0001$ ), end stage renal disease (aOR: $2.19 ; \mathrm{p}<0.0001$ ), systemic lupus erythematous (aOR: 3.76 ;

Review began $03 / 13 / 2021$ Review ended 04/15/2021 Published 04/24/2021

\section{(๑) Copyright 2021}

Patel et al. This is an open access article distributed under the terms of the Creative Commons Attribution License CC-BY 4.0., which permits unrestricted use, distribution, and reproduction in any medium, provided the original author and source are credited. $\mathrm{p}<0.0001$ ), polymyositis (aOR: $2.72 ; \mathrm{p}=0.0105$ ), ankylosis spondylosis (aOR: $2.42 ; \mathrm{p}=0.0082$ ), hypercoagulable state (aOR: 4.03; $\mathrm{p}<0.0001$ ), polyarteritis nodosa (aOR: $5.65 ; \mathrm{p}=0.0004)$, and fibromuscular dysplasia (aOR: $2.83 ; \mathrm{p}<0.0001)$.

\section{Conclusion}

There is an increasing trend in AIS prevalence over time among YA. Both traditional and non-traditional risk factors suggest that greater awareness is needed, with prevention strategies for AIS among young adults.

Categories: Internal Medicine, Neurology, Epidemiology/Public Health

Keywords: acute ischemic stroke, risk factors , young adults, nationwide inpatient sample (nis), ischemic cerebrovascular disease, hypercoagulable state, end stage renal disease, hiv, epilepsy, obesity

\section{Introduction}

Stroke is the second leading cause of death globally, and although it is most common in the elderly, a significant number of young adults (YA) suffer from it every year [1,2]. The risk of stroke increases with age, but can occur at any age. In 2009, 34\% of people hospitalized for stroke were less than 65 years old [3]. Approximately $5-10 \%$ of strokes occur in adults $<45$ years of age [4-8]. Despite considerable improvements in primary prevention, diagnostic workup, and treatment, stroke still remains a major cause of morbidity, serious physical and cognitive long-term disability, and loss in work-related productivity especially when it occurs in the younger population $[9,10]$. 
In a systematic review of stroke incidence in YA, the proportion of ischemic strokes ranged between 21.0\% and $77.9 \%$ in patients under 45 years of age with first-ever stroke [11]. There were 59,077 deaths in YA in the United States from 1989 through 2009 due to stroke, contributing to 2868 deaths per year on average with an average annual mortality rate among YA being 0.93 per 100,000 persons for intracerebral hemorrhage (ICH), 1.1 per 100,000 persons for subarachnoid hemorrhage (SAH), and 0.70 per 100,000 persons for ischemic stroke [12]. In a single-center study comparing characteristics of stroke between younger and older patients, there were significant differences in risk factors, etiology, and distribution of sex between these groups [13]. Edwards et al. from the Canadian Institute for Health Information Discharge Abstract Database $(n=26,366)$ described a higher hazard for recurrent stroke at one year (hazard ratio $\{\mathrm{HR}\}: 6.8$ ), at five years (HR: 5.1), stroke survivors had higher mortality and morbidity, and patients with TIA had a higher prevalence (31.5\%; 1789/5677) of an adverse event within the first five years [14].

Our study aimed to provide estimates on the burden of stroke among YA in the United States. We performed a comprehensive assessment to compare traditional and non-traditional risk factors and ischemic strokerelated mortality, morbidity, discharge disposition, disability, and risk of death among young adults (YA: 1845 years) vs. old adults (OA: >45 years) between 2003 and 2014.

\section{Materials And Methods}

Data were obtained from the Agency for Healthcare Research and Quality's Healthcare Cost and Utilization Project (HCUP) Nationwide Inpatient Sample (NIS) files from January 2003 to December 2014. The NIS is the largest publicly available all-payer inpatient care database in the United States and contains discharge-level data provided by states that participate in the HCUP (including a total of 46 states in 2011). This administrative dataset contains data on approximately eight million hospitalizations in 1000 hospitals that were chosen to approximate a $20 \%$ stratified sample of all US community hospitals, representing more than 95\% of the national population. Discharge weights are provided for each patient discharge record, which helps to obtain national estimates. Each hospitalization is treated as an individual entry in the database and is coded with one principal diagnosis, up to 24 secondary diagnoses, and 15 procedural diagnoses associated with that stay (detailed information on NIS is available at http://www.hcup-

us.ahrq.gov/db/nation/nis/nisdde.jsp). The NIS is a de-identified database, so informed consent or IRB approval was not needed for the study. The HCUP Data Use Agreement and training (HCUP-4Q28K90CU) for the data utilized in this study were obtained.

\section{Study population}

We used the ninth revision of the International Classification of Diseases, Ninth Revision, Clinical Modification (ICD-9-CM) codes to identify adult patients admitted with a primary diagnosis of AIS (ICD-9CM codes 433.01, 433.11, 433.21, 433.31, 433.81, 433.91, 434.01, 434.11, 434.91). These codes have been previously validated and are $35 \%$ sensitive, $99 \%$ specific, with $96 \%$ positive predictive value (PPV), and $79 \%$ negative predictive value for the diagnosis of ischemic stroke [15]. We used ICD-9-CM codes to identify traditional and non-traditional risk factors. Table 1 lists all ICD-9-CM codes that were used for this study. Age $<18$ years and admissions with missing data for age, sex, and race were excluded.

\section{Disease}

Infective endocarditis

Cardiomyopathy

Rheumatic fever

IHD

ASD

VSD

PDA

Rheumatoid arthritis

Ankylosing spondylitis

Psoriatic arthritis

SLE
ICD9-CM codes nationwide inpatient sample

421.0

425.1 Primary CM, 425.20 Obscure cardiomyopathy of Africa, 425.30 Endocardial fibroelastosis, 425.40 Other primary cardiomyopathies, 425.50 Alcoholic cardiomyopathy, 425.70 Nutritional and metabolic cardiomyopathy, 425.80-429.83 Cardiomyopathy in other diseases classified Takotsubo, 425.90, 674.5, 414.8 Secondary cardiomyopathy, unspecified Peripartum cardiomyopathy Ischemic

$390,391.9,391.1,391.8,391.2,391.0$

$410.00,410.01,410.02,410.10,410.11,410.12,410.20,410.21,410.22,410.30,410.31,410.32,410.40$, $410.41,410.42$, 410.50, 410.51, 410.52, 410.60, 410.61, 410.62, 410.70, 410.71, 410.72, 410.80, 410.81, 410.82 , 410.90, 410.91, 410.92, CAD: 414.00-414.07, old MI - 412

$745.5,745.61$

745.4

747.0

$714.0,714.1,714.2$

720.0

696.0

710.0 


\section{Cureus}

Scleroderma
Sjogren's syndrome
Polymyositis
Dermatomyositis
Hypercoagulable
disorders (factor V
Leiden mutation,
antiphospholipid
antibodies, protein S
deficiency, antithrombin
III deficiency

Polycythemia rubra

Pneumonia

Urinary tract infection

TB meningitis

Tuberculoma

Neurosyphilis

Cryptococcal meningitis

Seizure

CNS tumors

AVM brain
Moyamoya
Giant cell arteritis
PAN
Takayasu's disease
Thromboangiitis
obliterans
HIV
Fabry's

Fibromuscular dysplasia

Sickle cell disease

Pregnancy

Pregnancy-related
701.0

710.2

710.4

710.3

286.53, 289.81, 795.79

238.4

Viral - 480.0, 480.1, 480.2, 480.3, 480.8, 480.9, Pneumococcal- 481 Other - bacterial pneumonia- 482.0, 482.1, 482.2, 482.3, Strep - 482.30, 482.31, 482.32, 482.39, Staph - 482.40, 482.41, 482.42, 482.49, Other specified bacteria - 482.81, 482.82, 482.83, 482.84, 482.89, 482.9, 483.0, 483.1, 483.8486

599.0

013.00

013.20

094.0 Tabes dorsalis, 094.1 General paresis, 094.2 Syphilitic meningitis, 094.3 Asymptomatic neurosyphilis, 094.81 Syphilitic encephalitis, 094.82 Syphilitic parkinsonism, 094.83 Syphilitic disseminated, 094.84 Syphilitic optic atrophy, 094.85 Syphilitic retrobulbar neuritis, 094.86 Syphilitic acoustic neuritis, 094.87 Syphilitic ruptured cerebral aneurysm, 094.89 Other specified neurosyphilis, 094.9 Neurosyphilis, unspecified

\section{0}

345.01 generalized nonconvulsive epilepsy 345.0 nonconvulsive/absence 345.1 Gen convulsion 345.2 Petit mal status 345.3 Grand mal status 345.4 Partial epi w impairment of consciousness 345.5 partial epi w/o impairment of cons 780.3 Convulsion excluding epileptic convulsion \& of newborn 780.39 other convulsion 780.31 febrile convulsion 345.6 infantile spasms 345.81 Intractable epilepsy

191.0, Cerebrum, except lobes and ventricles 191.1, Frontal lobe. 191.2, Temporal lobe 191.3, Parietal lobe. 191.4, Occipital lobe. 191.5, Ventricles, 191.6, Cerebellum, 191.7, Brain stem. 191.8, Other parts of brain, 191.9, Brain unspecified and cranial fossa unspecified.

\subsection{1}

437.5

446.5

446.0

446.7

443.1

042, V08

272.7

447.8447 .3

282.60, 282.62 Hb SS with crisis $282.61 \mathrm{Hb}$ SS without crisis $282.63 \mathrm{SC} / \mathrm{HbC}$ w/o crisis $282.64 \mathrm{SC} / \mathrm{HbC} \mathrm{w}$ crisis 282.68 other SCD without crisis 282.69 other SCD with crisis 282.41 Sickle cell-thalassemia without crisis 282.42 Sickle cell-thalassemia with crisis

V22.0, V22.1, V22.2, V23.9

Hyperemesis 643.10, 643.11, 643.13 Preterm labor 644.00, 644.03, 644.10, 644.13, 644.20, 644.21 Antepartum hemorrhage, 641.10, 641.11, 641.13, 641.30, 641.31, 641.33, 641.80, 641.83, 641.90, 641.93 Preeclampsia and gestational hypertension $642.40,642.41,642.42,642.43,642.44,642.50,642.51,642.52$, $642.53,642.54,642.60,642.61,642.62,642.63,642.64,642.70,642.71,642.72,642.73,642.74,642.90$, 


\section{Cureus}

conditions/complications

91, 642.92, 642.93, 642.94, diabetes - $648.00,648.01,648.02,648.03,648.04,648.80,648.81,648.82$, 648.83, 648.84, Postpartum hemorrhage 666.00, 666.02, 666.04, 666.10, 666.12, 666.14, 666.20, 666.22, 666.24, 666.30, 666.32, 666.34, Puerperial septic thrombophlebitis: $670.30,670.32,670.34$

Alcohol $303.00,303.01,303.02,303.03303 .90,303.91,303.92,303.93305 .0$

substance abuse $305.90,305.20,305.21,305.22,305.23,305.30,305.31,305.32,305.33,305.40,305.41,305.42,305.43$ 305.50, 305.51, 305.52, 305.53 305.60, 305.61, 305.62, 305.63 305.70, 305.71, 305.72, 305.73 305.80, $305.81,305.82,305.83305 .90,305.91,305.392,305.93$

Smoking

305.1, V15.82

Hypertension 401.0, 401.9, Complications - 402.00, 402.10, 402.90, 403.00, 403.10, 403.90, 404.00, 404.10, 404.90, 404.01, 404.11, 404.91, 404.93, 404.13, 404.93

$250.00,250.01,250.02,250.03,250.10,250.11,250.12,250.13,250.20,250.21,250.22,250.23,250.30$,

DM $250.31,250.32$, 250.33, 250.40, 250.41, 250.42, 250.43, 250.50, 250.51, 250.52, 250.53, 250.60, 250.61, $250.62,250.63,250.70,250.71,250.72,250.73$,

A.fib 427.31

Hypercholesterolemia
$272.0,272.1,272.2$

\section{TABLE 1: ICD-9-CM codes used in this analysis}

ICD9-CM: International Classification of Diseases, Ninth Revision, Clinical Modification; IHD: ischemic heart diseases; ASD: atrial septal disease; VSD: ventricular septal disease; PDA: patent ductus arteriosus; SLE: systemic lupus erythematosus; CNS: central nervous system; DM: diabetes mellitus; A-fib: atrial fibrillation; Hb SS: homozygous SCD patients; SC/HbC: sickle cell-hemoglobin C; SCD: sickle cell disease

\section{Patient and hospital characteristics}

Patient characteristics of interest were age, sex, race, insurance status, and concomitant diagnoses as defined above. The race was defined by white (referent), African American, Hispanic, Asian or Pacific Islander, and Native American. Insurance status was defined by Medicare (referent), Medicaid, Private Insurance, and Other/Self-pay/No charge. We defined the severity of co-morbid conditions using Deyo's modification of the Charlson Comorbidity Index (CCI) (Table 2). 


\section{Cureus}

\begin{tabular}{|c|c|c|}
\hline Condition & ICD-9-CM codes & Charlson score \\
\hline Myocardial infarction & $410-410.9$ & 1 \\
\hline Congestive heart failure & $428-428.9$ & 1 \\
\hline Peripheral vascular disease & $433.9,441-441.9,785.4, \mathrm{~V} 43.4$ & 1 \\
\hline Cerebrovascular disease & $430-438$ & 1 \\
\hline Dementia & $290-290.9$ & 1 \\
\hline Chronic pulmonary disease & $490-496,500-505,506.4$ & 1 \\
\hline Rheumatologic disease & $710.0,710.1,710.4,714.0-714.2,714.81,725$ & 1 \\
\hline Peptic ulcer disease & $531-534.9$ & 1 \\
\hline Mild liver disease & $571.2,571.5,571.6,571.4-571.49$ & 1 \\
\hline Diabetes & $250-250.3,250.7$ & 1 \\
\hline Diabetes with chronic complications & 250.4-250.6 & 2 \\
\hline Hemiplegia or paraplegia & $344.1,342-342.9$ & 2 \\
\hline Renal disease & $582-582.9,583-583.7,585,586,588-588.9$ & 2 \\
\hline Any malignancy including leukemia and lymphoma & $140-172.9,174-195.8,200-208.9$ & 2 \\
\hline Moderate or severe liver disease & $572.2-572.8$ & 3 \\
\hline Metastatic solid tumor & 196-199.1 & 6 \\
\hline AIDS & 042-044.9 & 6 \\
\hline
\end{tabular}

\section{TABLE 2: Deyo's modification of CCI}

ICD9-CM: International Classification of Diseases, Ninth Revision, Clinical Modification; CCI: Charlson Comorbidity Index

\section{Outcomes}

Our primary interest was to compare the prevalence of traditional and non-traditional risk factors of AIS among YA (18-45 years) and OA (>45 years). The secondary interest was to compare outcomes of AIS in YA and OA. The outcomes were all-cause mortality during hospitalization, morbidity (length of stay $>10$ days [>90th percentile of AIS hospitalization\} and discharge to non-home \{transfer to short-term hospital, skilled nursing facility, intermediate care facility, or home health care\}), discharge disposition (discharge to home vs. non-home), All Patients Refined Diagnosis Related Groups (APR-DRG) risk of mortality, APR-DRG severity of illness (disability), length of stay (LoS), and cost of hospitalization [16]. APR-DRGs were assigned using software developed by 3M Health Information Systems, where score 1 indicates minor loss of function, 2-moderate, 3-major, 4-extreme loss of function or likelihood of death. APR-DRG coding system used in this study to assess the risk of mortality and severity of illness is externally validated. It is a reliable method with accurate and consistent results and is widely used by hospitals, consumers, payers, and regulators $[17,18]$.

\section{Statistical analysis}

All statistical analyses were performed using the weighted survey methods in Statistical Analysis System (SAS) Version 9.4 (SAS Institute Inc., Cary, NC). Weighted values of patient-level observations were generated to produce a nationally representative estimate of the entire US population of hospitalized patients. Univariate analysis of differences between categorical variables (including demographics, comorbidities, risk factors, and concurrent conditions) and outcomes was tested using the chi-square test and analysis of differences between continuous variables (LoS and cost of hospitalization) was tested using unpaired student's t-test. Among AIS hospitalizations, the prevalence and mortality trends from 2003 to 2014 for YA and OA were tested and plotted using the Jonckheere trend test.

In order to examine the relationship of age groups (YA vs. OA) with AIS-related risk factors and the relationship of age groups with AIS-related outcomes, we used mixed-effects multivariable survey logistic regression models. The models were weighted and adjusted for demographics (age, sex, race), patient-level hospitalization variables (admission day, primary payer, admission type, median household income category), hospital-level variables (hospital region, teaching versus nonteaching hospital, hospital bed size), 
comorbidities, traditional and non-traditional risk factors, and CCI in order to estimate the adjusted odds ratio (aOR) and 95\% confidence interval (CI). Common conditions covered as risk factors and CCIs were adjusted only once in order to avoid over-adjustment. For each model, the c-index was calculated. All statistical tests used were two-sided, and $\mathrm{p}<0.05$ was deemed statistically significant. No statistical power calculation was conducted prior to the study.

\section{Results}

We have described prevalence trends and characteristics of AIS. We have also compared demographics, patient and hospital characteristics, comorbidities, and outcomes of AIS amongst YA and OA below.

\section{Disease hospitalizations}

There were 4,224,924 hospitalizations due to AIS from 2003 to 2014 after excluding patients with age $<18$ years and admissions with missing data for age, sex, and race (Figure 1). Out of 4,224,924 AIS hospitalizations, 198,378 (4.7\%) were YA ( $\leqslant 45$ years) and 4,026,546 (95.3\%) were OA. As shown in Figure 2, the percentage of YA among AIS hospitalizations increased from 4.36\% in 2003 to $4.7 \%$ in 2014. (pTrend $<0.0001)$

Nationwide inpatient sample database from the year 2003-2014

$\downarrow$

Patients with Acute Ischemic Stroke (AIS) were identified by using ICD-9-CM codes [N=5,048,917 (Unweighted $\mathbf{N}=\mathbf{1 , 0 2 4 , 3 6 4 ) ]}$

$$
\downarrow
$$

Age $<18$ years and admissions with missing data for age, gender, and race were excluded<smiles>C1C2CC1C2</smiles>

Final AIS study population $(\mathrm{N}=4,224,924)$

$\downarrow$

Young adults (18-45 years) $[\mathrm{N}=198378(4.7 \%)]$ and old patients $(>45$ years) $[\mathrm{N}=4026546$ $(95.3 \%)]$ were identified

$\downarrow$

AIS prevalence, trend, mortality-trend between young adults and old patients were identified

$\downarrow$

The prevalence and odds (association) of traditional and non-traditional risk factors of AIS among YA (18-45 years) and $\mathrm{OA}(>45$ years) were evaluated using univariate analysis and multivariate survey logistic regression analysis $\downarrow$

The outcomes (mortality, morbidity, discharge disposition, risk of mortality, disability, hospitalization length, and cost) of AIS among YA and OA were evaluated using univariate analysis and multivariate survey logistic regression analysis

FIGURE 1: Flowchart detailing cohort selection and modeling analysis of outcomes

ICD9-CM: International Classification of Diseases, Ninth Revision, Clinical Modification; AIS: acute ischemic stroke; YA: young adults; OA: old adults 


\section{Cureus}

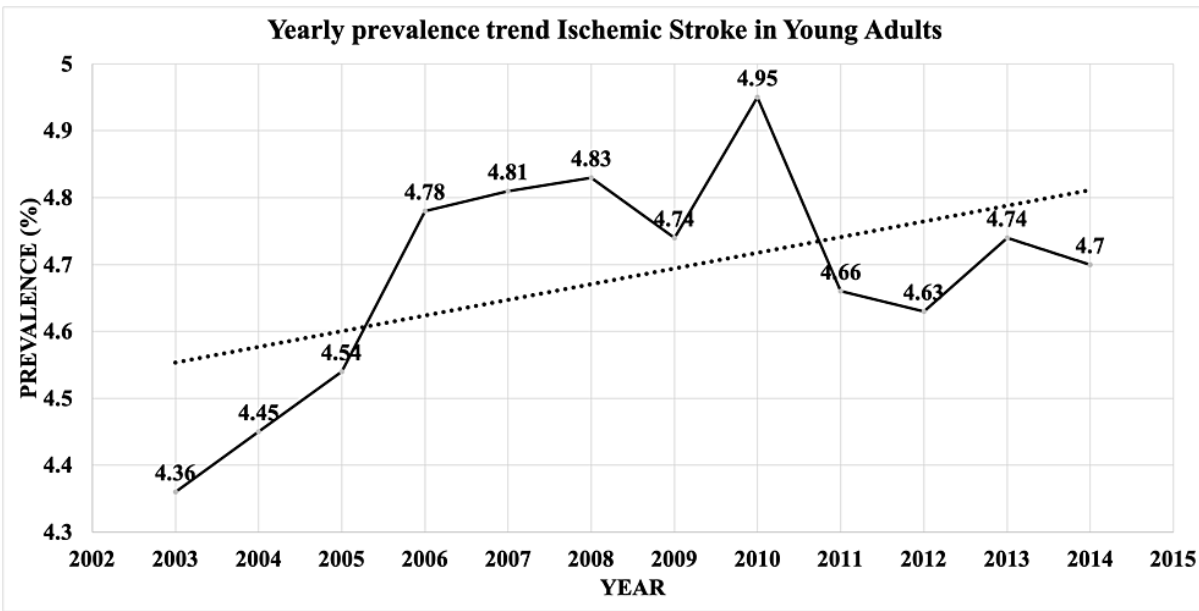

FIGURE 2: Yearly prevalence trend of AIS in Young adults

AIS: acute ischemic stroke

Demographics, patient and hospital characteristics, and comorbidities

There was a higher proportion of females among OA with AIS than YA (53.1\% vs. 48.5\%; p<0.0001) (Table 3). There was a higher proportion of African Americans (29.95\% vs. 16.14\%; $\mathrm{p}<0.0001)$ and Hispanics $(12.17 \%$ vs. $7.35 \%$; $<0.0001)$ among YA. Utilization of recombinant tissue plasminogen activator (6.32\% vs. $4.34 \%$; $\mathrm{p}<0.0001)$ and endovascular mechanical thrombectomy $(1.22 \%$ vs. $0.58 \%$; $p<0.0001)$ were higher amongst YA with AIS compared to OA with AIS. OA with AIS had a higher prevalence of current long-term use of aspirin therapy compared to YA ( $8.79 \%$ vs. $5.12 \%$; $\mathrm{p}<0.0001)$. Several co-morbidities like chronic blood loss anemia ( $0.7 \%$ vs. $0.45 \%$; $p<0.0001)$, liver disease ( $1.34 \%$ vs. $1.06 \%$; $<<0.0001)$, paralysis $(5.48 \%$ vs. $3.7 \%$; $p<0.0001)$, psychosis $(4.57 \%$ vs. $2.91 \%$; $\mathrm{p}<0.0001)$ and chronic neurologic disorders $(0.88 \%$ vs. $0.48 \%$; $p<0.0001)$ were higher among YA than OA.

\begin{tabular}{|c|c|c|c|c|}
\hline & $\begin{array}{l}\text { Young adults }(18-45 \\
\text { years) }\end{array}$ & $\begin{array}{l}\text { Older adults }(>45 \\
\text { years) }\end{array}$ & Total & p-Value \\
\hline AIS hospitalizations (\%) & $198,378(4.7)$ & $4,026,546$ (95.3) & $4,224,924(100)$ & \\
\hline \multicolumn{5}{|l|}{ Demographics of patients } \\
\hline Gender (\%) & & & & $<0.0001$ \\
\hline Female & $96,233(48.51)$ & 2,138,020 (53.1) & $\begin{array}{l}2,234,253 \\
(52.88)\end{array}$ & \\
\hline Male & 102,145 (51.49) & 1,888,457 (46.9) & $\begin{array}{l}1,990,602 \\
(47.12)\end{array}$ & \\
\hline Race (\%) & & & & $<0.0001$ \\
\hline White & 104,485 (54.54) & 2,881,643 (73.4) & $\begin{array}{l}2,986,128 \\
(72.53)\end{array}$ & \\
\hline African American & $57,382(29.95)$ & 633,708 (16.14) & $691,090(16.78)$ & \\
\hline Hispanic & $23,310(12.17)$ & $288,462(7.35)$ & $311,772(7.57)$ & \\
\hline Asian or Pacific Islander & 5158 (2.69) & $103,386(2.63)$ & $108,544(2.64)$ & \\
\hline Native American & $1233(0.64)$ & $18,600(0.47)$ & $19,833(0.48)$ & \\
\hline \multicolumn{5}{|l|}{ Characteristics of patients } \\
\hline $\begin{array}{l}\text { Median household income category for patient's ZIP } \\
\text { code }(\%)^{\star}\end{array}$ & & & & $<0.0001$ \\
\hline 0-25th percentile & 66,609 (34.44) & 1,175,425 (29.82) & $\begin{array}{l}1,242,034 \\
(30.04)\end{array}$ & \\
\hline
\end{tabular}




\section{Cureus}

\begin{tabular}{|c|c|c|c|c|}
\hline 26-50th percentile & 49,480 (25.58) & $1,015,750(25.77)$ & $\begin{array}{l}1,065,230 \\
(25.76)\end{array}$ & \\
\hline 51-75th percentile & 42.785 (22.12) & $920,665(23.36)$ & $963,450(23.3)$ & \\
\hline 76-100th percentile & $34,548(17.86)$ & $829,372(21.04)$ & $863,920(20.89)$ & \\
\hline Primary payer (\%) & & & & $<0.0001$ \\
\hline Medicare & 18,906 (9.56) & 2,804,887 (69.78) & $\begin{array}{l}2,823,793 \\
(66.95)\end{array}$ & \\
\hline Medicaid & $46,516(23.52)$ & $239,588(5.96)$ & $286,104(6.78)$ & \\
\hline Private insurance & $87,892(44.44)$ & $711,337(17.7)$ & $799,229(18.95)$ & \\
\hline Other/self-pay/no charge & $44,470(22.48)$ & $263,983(6.57)$ & $308,453(7.31)$ & \\
\hline Admission type (\%) & & & & $<0.0001$ \\
\hline Non- elective & 190,181 (96.06) & 3,833,377 (95.41) & $\begin{array}{l}4,023,557 \\
(95.44)\end{array}$ & \\
\hline Elective & $7806(3.94)$ & $184,578(4.59)$ & $192,384(4.56)$ & \\
\hline Admission day (\%) & & & & 0.0018 \\
\hline Weekday & $148,268(74.74)$ & 2,996,820 (74.43) & $\begin{array}{l}3,145,089 \\
(74.44)\end{array}$ & \\
\hline Weekend & $50,110(25.26)$ & $1,029,725(25.57)$ & $\begin{array}{l}1,079,835 \\
(25.56)\end{array}$ & \\
\hline \multicolumn{5}{|l|}{ Characteristics of hospitals } \\
\hline Bedsize of hospital $(\%)^{\star \star}$ & & & & $<0.0001$ \\
\hline Small & $17,009(8.63)$ & $481,564(12.01)$ & $498,573(11.85)$ & \\
\hline Medium & $47,805(24.26)$ & $1,030,840(25.71)$ & $\begin{array}{l}1,078,644 \\
(25.64)\end{array}$ & \\
\hline Large & $132,226(67.11)$ & 2,497,587 (62.28) & $\begin{array}{l}2,629,813 \\
(62.51)\end{array}$ & \\
\hline Hospital location \& teaching status (\%) & & & & $<0.0001$ \\
\hline Rural & $14,062(7.14)$ & 481,027 (12) & 495,089 (11.77) & \\
\hline Urban non-teaching & 72,265 (36.68) & $1,710,411(42.65)$ & $\begin{array}{l}1,782,676 \\
(42.37)\end{array}$ & \\
\hline Urban teaching & 110,712 (56.19) & 1,818,553 (45.35) & $\begin{array}{l}1,929,265 \\
(45.86)\end{array}$ & \\
\hline Hospital region (\%) & & & & $<0.0001$ \\
\hline Northeast & $37,829(19.07)$ & $858,527(21.32)$ & $896,356(21.22)$ & \\
\hline Midwest & $32,990(16.63)$ & $697,196(17.31)$ & $730,186(17.28)$ & \\
\hline South & 90,734 (45.74) & $1,719,665(42.71)$ & $\begin{array}{l}1,810,399 \\
(42.85)\end{array}$ & \\
\hline West & $36,826(18.56)$ & $751,158(18.66)$ & $787,983(18.65)$ & \\
\hline \multicolumn{5}{|l|}{ Stroke related medications (\%) } \\
\hline Current long-term use of Aspirin therapy & $10,165(5.12)$ & $353,886(8.79)$ & $364,051(8.62)$ & $<0.0001$ \\
\hline Use of recombinant tissue plasminogen activator (rtPA) & $12,534(6.32)$ & $174,855(4.34)$ & $187,388(4.44)$ & $<0.0001$ \\
\hline Use of endovascular mechanical thrombectomy & $2413(1.22)$ & $23,411(0.58)$ & $25,824(0.61)$ & $<0.0001$ \\
\hline \multicolumn{5}{|l|}{ Comorbidities of patients (\%) } \\
\hline Deficiency anemias & 19,652 (9.94) & 467,108 (11.65) & 486,759 (11.57) & $<0.0001$ \\
\hline
\end{tabular}




\section{Cureus}

\begin{tabular}{|c|c|c|c|c|}
\hline Rheumatoid arthritis/collagen vascular diseases & $5321(2.69)$ & $94,806(2.37)$ & $100,127(2.38)$ & $<0.0001$ \\
\hline Chronic blood loss anemia & $1381(0.7)$ & $17,913(0.45)$ & $19294.6(0.46)$ & $<0.0001$ \\
\hline Congestive heart failure & $12,425(6.28)$ & $578,171(14.43)$ & $590,596(14.04)$ & $<0.0001$ \\
\hline Chronic pulmonary disease & $17,442(8.82)$ & $602,466(15.03)$ & 619,908 (14.74) & $<0.0001$ \\
\hline Hypothyroidism & 8535 (4.32) & $514,168(12.83)$ & $522,703(12.43)$ & $<0.0001$ \\
\hline Liver disease & $2654(1.34)$ & $42,508(1.06)$ & $45,162(1.07)$ & $<0.0001$ \\
\hline Lymphoma & $632(0.32)$ & $20,836(0.52)$ & $21,468(0.51)$ & $<0.0001$ \\
\hline Fluid and electrolyte disorders & 30,624 (15.49) & $800,738(19.98)$ & $831,362(19.77)$ & $<0.0001$ \\
\hline Metastatic cancer & $1116(0.56)$ & $58,488(1.46)$ & $59,604(1.42)$ & $<0.0001$ \\
\hline Paralysis & $10,826(5.48)$ & $148,241(3.7)$ & $159,067(3.78)$ & $<0.0001$ \\
\hline Psychoses & $9036(4.57)$ & $116,737(2.91)$ & $125,774(2.99)$ & $<0.0001$ \\
\hline Peptic ulcer disease excluding bleeding & $25(0.01)$ & $1416(0.04)$ & $1441(0.03)$ & $<0.0001$ \\
\hline Valvular disease & $13,434(6.8)$ & $413,319(10.31)$ & 426,752 (10.15) & $<0.0001$ \\
\hline Welght loss & $3289(1.66)$ & $125,944(3.14)$ & $129,233(3.07)$ & $<0.0001$ \\
\hline Pulmonary circulation disorders & $3317(1.68)$ & $115,370(2.88)$ & $118,687(2.82)$ & $<0.0001$ \\
\hline Peripheral vascular disease & $14,352(7.26)$ & $359,101(8.96)$ & $373,453(8.88)$ & $<0.0001$ \\
\hline Coagulopathy & $5614(2.84)$ & $110,457(2.76)$ & $116,071(2.76)$ & 0.0267 \\
\hline Solid tumor without metastasis & $884(0.45)$ & 70,634 (1.76) & $71,518(1.7)$ & $<0.0001$ \\
\hline Depression & $18,818(9.52)$ & $370,045(9.23)$ & $388,863(9.25)$ & $<0.0001$ \\
\hline Other neurological disorders & $1743(0.88)$ & $19,275(0.48)$ & $21,018(0.5)$ & $<0.0001$ \\
\hline \multicolumn{5}{|l|}{ Concurrent conditions or risk factors (\%) } \\
\hline Diabetes & $49,279(24.84)$ & $1,394,654(34.64)$ & $\begin{array}{l}1,443,933 \\
(34.18)\end{array}$ & $<0.0001$ \\
\hline $\begin{array}{l}\text { Hypertension (combined uncomplicated and } \\
\text { complicated) }\end{array}$ & 109,951 (55.42) & $3,246,458(80.63)$ & $\begin{array}{l}3,356,409 \\
(79.44)\end{array}$ & $<0.0001$ \\
\hline Obesity & 31,797 (16.03) & $306,293(7.61)$ & $338,091(8)$ & $<0.0001$ \\
\hline Hypercholesterolemia & $16,298(8.22)$ & 435,347 (10.81) & $451,645(10.69)$ & $<0.0001$ \\
\hline Drug abuse/dependence & 21,714 (10.95) & $67,645(1.68)$ & $89,359(2.12)$ & $<0.0001$ \\
\hline Alcohol abuse/dependence & $13,067(6.59)$ & $149,261(3.71)$ & $162,328(3.84)$ & $<0.0001$ \\
\hline Past history of smoking & $9262(4.67)$ & $379,316(9.42)$ & $388,579(9.2)$ & $<0.0001$ \\
\hline Current tobacco dependence & $61,720(31.11)$ & $574,046(14.26)$ & 635,766 (15.05) & $<0.0001$ \\
\hline Cardiac diseases & $45,679(23.03)$ & $1,896,107(47.09)$ & $\begin{array}{l}1,941,786 \\
(45.96)\end{array}$ & $<0.0001$ \\
\hline Ischemic heart disease & $17,934(9.04)$ & $1,147,546(28.5)$ & $\begin{array}{l}1,165,481 \\
(27.59)\end{array}$ & $<0.0001$ \\
\hline Infective endocarditis & $965(0.49)$ & $6262(0.16)$ & $7227(0.17)$ & $<0.0001$ \\
\hline Atrial Fibrillation & 5926 (2.99) & $947,502(23.53)$ & $953,428(22.57)$ & $<0.0001$ \\
\hline Cardiomyopathy & $10,203(5.14)$ & $144,888(3.6)$ & $155,091(3.67)$ & $<0.0001$ \\
\hline Rheumatic fever & $50(0.03)$ & $368(0.01)$ & $418(0.01)$ & $<0.0001$ \\
\hline Rheumatoid heart disease & 3859 (1.95) & $133,365(3.31)$ & $137,224(3.25)$ & $<0.0001$ \\
\hline ial septal disease & $15,181(7.65)$ & 69,403 (1.72) & $84,584(2)$ & $<0.0001$ \\
\hline
\end{tabular}




\section{Cureus}

\begin{tabular}{|c|c|c|c|c|}
\hline Ventricular septal disease & $230(0.12)$ & $550(0.01)$ & $781(0.02)$ & $<0.0001$ \\
\hline Patent ductus arteriosus & $37(0.02)$ & $249(0.01)$ & $286(0.01)$ & $<0.0001$ \\
\hline Infectious diseases & $16,980(8.56)$ & 626,884 (15.57) & 643,864 (15.24) & $<0.0001$ \\
\hline Urinary tract infection & $11,209(5.65)$ & $509,319(12.65)$ & 520,528 (12.32) & $<0.0001$ \\
\hline HIV infection & $2425(1.22)$ & $5679(0.14)$ & $8104(0.19)$ & $<0.0001$ \\
\hline Pneumonia & $4179(2.11)$ & 144,646 (3.59) & 148,825 (3.52) & $<0.0001$ \\
\hline Neurosyphilis & $133(0.07)$ & $1857(0.05)$ & $1989(0.05)$ & $<0.0001$ \\
\hline CNS tuberculosis & $16(0.01)$ & $25(0)$ & $41(0)$ & $<0.0001$ \\
\hline Meningitis & $75(0.04)$ & $276(0.01)$ & $352(0.01)$ & $<0.0001$ \\
\hline CMV encephalitis & $57(0.03)$ & $202(0.01)$ & $259(0.01)$ & $<0.0001$ \\
\hline Toxoplasmosis & $<10$ & 0 & $<10$ & $<0.0001$ \\
\hline CNS lymphoma & $<10$ & 84 & 94 & 0.0142 \\
\hline Progressive multifocal encephalopathy & $43(0.02)$ & 98 & 141 & $<0.0001$ \\
\hline Non-infective CNS diseases & $30,110(15.18)$ & 618,165 (15.35) & 648,274 (15.34) & 0.0355 \\
\hline Brain tumors & $336(0.17)$ & $2442(0.06)$ & $2778(0.07)$ & $<0.0001$ \\
\hline Epilepsy & $16,641(8.39)$ & $235,995(5.86)$ & $252,636(5.98)$ & $<0.0001$ \\
\hline Hemorrhagic stroke & 3165 (1.6) & $67,054(1.67)$ & $70,219(1.66)$ & 0.0172 \\
\hline Arterial-venous malformation & $576(0.29)$ & $3919(0.1)$ & $4496(0.11)$ & $<0.0001$ \\
\hline History of transient ischemic attack & $11,304(5.7)$ & $338,425(8.4)$ & $349,728(8.28)$ & $<0.0001$ \\
\hline Traumatic brain injury & $217(0.11)$ & $7861(0.2)$ & $8078(0.19)$ & $<0.0001$ \\
\hline Renal diseases & 19,855 (10.01) & 652,927 (16.22) & 672,783 (15.92) & $<0.0001$ \\
\hline Chronic kidney diseases & $7427(3.74)$ & $342,859(8.51)$ & $350,286(8.29)$ & $<0.0001$ \\
\hline Acute renal failure & $9328(4.7)$ & $279,229(6.93)$ & $288,557(6.83)$ & $<0.0001$ \\
\hline End-stage renal disease & 3787 (1.91) & $61,935(1.54)$ & $65,722(1.56)$ & $<0.0001$ \\
\hline Connective tissue diseases & $5121(2.58)$ & $79,066(1.96)$ & $84,187(1.99)$ & $<0.0001$ \\
\hline Systemic lupus erythematous & $3959(2)$ & $14,170(0.35)$ & $18,129(0.43)$ & $<0.0001$ \\
\hline Scleroderma & $20(0.01)$ & $215(0.01)$ & $235(0.01)$ & 0.0051 \\
\hline Systemic sclerosis & $230(0.12)$ & $3995(0.1)$ & $4226(0.1)$ & 0.0203 \\
\hline Rheumatoid arthritis & $1022(0.51)$ & $59,416(1.48)$ & $60,438(1.43)$ & $<0.0001$ \\
\hline Polymyositis & $60(0.03)$ & $770(0.02)$ & $830(0.02)$ & 0.0007 \\
\hline Dermatomyositis & $25(0.01)$ & $394(0.01)$ & $419(0.01)$ & 0.1855 \\
\hline Ankylosis spondylosis & $91(0.05)$ & $971(0.02)$ & $1063(0.03)$ & $<0.0001$ \\
\hline Psoriatic arthritis & $124(0.06)$ & $2469(0.06)$ & $2593(0.06)$ & 0.8566 \\
\hline Coagulopathy & $9792(4.94)$ & $34,353(0.85)$ & $44,145(1.04)$ & $<0.0001$ \\
\hline Hypercoagulable state & $9057(4.57)$ & $22,625(0.56)$ & $31,682(0.75)$ & $<0.0001$ \\
\hline Polycythemia vera & $784(0.4)$ & $11,921(0.3)$ & $12,705(0.3)$ & $<0.0001$ \\
\hline Vasculitis & $222(0.11)$ & $6278(0.16)$ & $6500(0.15)$ & $<0.0001$ \\
\hline Giant cell arteritis & $30(0.01)$ & $5576(0.14)$ & $5605(0.13)$ & $<0.0001$ \\
\hline Polyarteritis nodosa & $47(0.02)$ & $288(0.01)$ & $335(0.01)$ & $<0.0001$ \\
\hline Takayasu disease & $85(0.04)$ & $166(0)$ & $250(0.01)$ & $<0.0001$ \\
\hline
\end{tabular}




\section{Cureus}

$\begin{array}{lllll}\text { Thromboangiitis obliterans } & 66(0.03) & 248(0.01) & 314(0.01) & <0.0001 \\ \text { Amyloidosis } & 19(0.01) & 3957(0.1) & 3976(0.09) & <0.0001 \\ \text { Sickle cell disease } & 894(0.45) & 1772(0.04) & 2666(0.06) & <0.0001 \\ \text { Moya-moya } & 1270(0.64) & 1020(0.03) & 2291(0.05) & <0.0001 \\ \text { Fibromuscular dysplasia } & 560(0.28) & 2957(0.07) & 3517(0.08) & <0.0001\end{array}$

\section{TABLE 3: Characteristics of AIS hospitalizations stratified by age group}

Percentage in brackets are column \% indicates the direct comparison between young adults vs. older adults among patients with AIS.

${ }^{\star}$ This represents a quartile classification of the estimated median household income of residents in the patient's ZIP code.

**Bedsize of hospital indicates the number of hospital beds which varies depends on hospital location (rural/urban), teaching status (teaching/nonteaching), and region (Northeast/Midwest/Southern/Western).

CMV: cytomegalovirus; CNS: central nervous system; AIS: acute ischemic stroke

\section{Primary outcome}

The prevalence of obesity (16.03\% vs. $7.61 \%$; p<0.0001), drug abuse (10.95\% vs. $1.68 \%$; $<<0.0001)$, alcohol abuse (6.59\% vs. $3.71 \%$; $<<0.0001)$, tobacco dependence (31.11\% vs. $14.26 \%$; $<0.0001)$, cardiomyopathy (5.14\% vs. 3.6\%; p<0.0001), atrial septal disease (7.56\% vs. $1.72 \%$; $\mathrm{p}<0.0001)$, epilepsy (8.39\% vs. 5.36\%; $\mathrm{p}<0.0001)$, and hypercoagulable state $(4.57 \%$ vs. $0.56 \%$; $<<0.0001)$ were higher among YA in compare to OA.

The OA with AIS had higher prevalence of diabetes (34.64\% vs. $24.84 \%$; $\mathrm{p}<0.0001)$, hypertension (80.63\% vs $55.42 \%$; $\mathrm{p}<0.0001)$, hypercholesterolemia/triglyceridemia (10.81\% vs. $8.22 \%$; $\mathrm{p}<0.0001)$ history of smoking (9.42\% vs. $4.67 \%$; $p<0.0001)$, ischemic heart disease (28.5\% vs. $9.04 \%$; $<<0.0001)$, atrial fibrillation $(23.53 \%$ vs. $2.99 \%$; $<<0.0001)$, rheumatoid heart disease $(3.31 \%$ vs. $1.95 \%$; $<<0.0001)$, urinary tract infection $(12.65 \%$ vs. $5.65 \%$; $\mathrm{p}<0.0001)$, pneumonia (3.59\% vs. $2.11 \%$; $\mathrm{p}<0.0001)$, history of transient ischemic attack (8.4\% vs. $5.7 \%$; $<0.0001)$, chronic kidney diseases $(8.51 \%$ vs. $3.74 \%$; $<<0.0001)$, and acute renal failure $(6.93 \%$ vs. $4.7 \% ; \mathrm{p}<0.0001)$.

\section{Multivariable regression model derivation for the age-group specific risk factors}

Table 4 shows multivariable models evaluating the odds of risk factors of AIS among YA and OA population. The obesity (aOR: $2.26 ; \mathrm{p}<0.0001$ ), drug abuse (aOR: $2.56 ; \mathrm{p}<0.0001$ ), past history of smoking (aOR: 1.20 ; $\mathrm{p}<0.0001$ ), infective endocarditis (aOR: $2.08 ; \mathrm{p}<0.0001$ ), cardiomyopathy (aOR: $2.11 ; \mathrm{p}<0.0001$ ), rheumatic fever (aOR: 4.27; $\mathrm{p}=0.0014$ ), atrial septal disease (aOR: 2.46 ; $\mathrm{p}<0.0001)$, ventricular septal disease (aOR: 4.99; $\mathrm{p}<0.0001$ ), HIV infection (aOR: 4.36; $\mathrm{p}<0.0001$ ), brain tumors (aOR: 7.89; $\mathrm{p}<0.0001$ ), epilepsy (aOR: 1.43; $\mathrm{p}<0.0001)$, arterial-venous malformation (aOR: $1.81 ; \mathrm{p}<0.0001)$, end-stage renal disease (aOR: 2.19 ; $\mathrm{p}<0.0001$ ), systemic lupus erythematous (aOR: 3.76 ; $\mathrm{p}<0.0001$ ), polymyositis (aOR: $2.72 ; \mathrm{p}=0.0105$ ), ankylosis spondylosis (aOR: $2.42 ; \mathrm{p}=0.0082$ ), hypercoagulable state (aOR: $4.03 ; \mathrm{p}<0.0001)$, polyarteritis nodosa (aOR: $5.65 ; \mathrm{p}=0.0004$ ), and fibromuscular dysplasia (aOR: 2.83 ; $\mathrm{p}<0.0001$ ) were significantly associated with YA population with AIS.

\begin{tabular}{|c|c|c|c|c|c|c|}
\hline & \multicolumn{3}{|c|}{$\begin{array}{l}\text { Association of risk factors with young } \\
\text { adults }\end{array}$} & \multicolumn{3}{|c|}{$\begin{array}{l}\text { Association of risk factors with old } \\
\text { adults }\end{array}$} \\
\hline & aOR & 95\% Cl (LL-UL) & $p$-Value & $\mathrm{aOR}$ & 95\% Cl (LL-UL) & $p$-Value \\
\hline \multicolumn{7}{|l|}{ Gender } \\
\hline Female & \multicolumn{2}{|c|}{ Reference } & $<0.0001$ & \multicolumn{2}{|c|}{ Reference } & $<0.0001$ \\
\hline Male & 0.89 & $0.87-0.91$ & & 1.12 & $1.09-1.15$ & \\
\hline \multicolumn{7}{|l|}{ Race } \\
\hline White & \multicolumn{2}{|c|}{ Reference } & $<0.0001$ & & & $<0.0001$ \\
\hline African American & 1.53 & $1.48-1.57$ & & 0.66 & $0.64-0.68$ & \\
\hline
\end{tabular}




\section{Cureus}

\begin{tabular}{|c|c|c|c|c|c|c|}
\hline Hispanic & 1.47 & $1.42-1.53$ & & 0.68 & $0.65-0.71$ & \\
\hline Asian or Pacific Islander & 1.08 & 1.00-1.16 & & 0.93 & $0.87-1.00$ & \\
\hline Native American & 1.27 & $1.08-1.48$ & & 0.79 & $0.67-0.93$ & \\
\hline \multicolumn{7}{|c|}{ Median household income category for patient's ZIP code } \\
\hline $0-25$ th percentile & \multicolumn{2}{|c|}{ Reference } & 0.0002 & \multicolumn{2}{|c|}{ Reference } & 0.0002 \\
\hline 26-50th percentile & 1.05 & 1.02-1.08 & & 0.96 & 0.93-0.99 & \\
\hline 51-75th percentile & 1.01 & $0.98-1.04$ & & 0.99 & $0.96-1.03$ & \\
\hline 76-100th percentile & 0.97 & 0.93-1.01 & & 1.03 & $1.00-1.07$ & \\
\hline \multicolumn{7}{|l|}{ Primary Payer } \\
\hline Medicare & \multicolumn{2}{|c|}{ Reference } & $<0.0001$ & \multicolumn{2}{|c|}{ Reference } & $<0.0001$ \\
\hline Medicaid & 15.69 & 14.99-16.42 & & 0.06 & $0.06-0.07$ & \\
\hline Private insurance & 11.10 & $10.69-11.53$ & & 0.06 & 0.09-0.09 & \\
\hline Other/self-pay/no charge & 13.70 & 13.11-14.32 & & 0.07 & $0.07-0.08$ & \\
\hline \multicolumn{7}{|l|}{ Admission type } \\
\hline Non-elective & \multicolumn{2}{|c|}{ Reference } & 0.0003 & \multicolumn{2}{|c|}{ Reference } & 0.0003 \\
\hline Elective & 0.90 & $0.84-0.95$ & & 1.12 & 1.05-1.19 & \\
\hline \multicolumn{7}{|l|}{ Admission day } \\
\hline Weekday & \multicolumn{2}{|c|}{ Reference } & 0.1418 & \multicolumn{2}{|c|}{ Reference } & 0.1418 \\
\hline Weekend & 0.98 & $0.95-1.01$ & & 1.02 & 0.99-1.05 & \\
\hline \multicolumn{7}{|l|}{ Bedsize of hospital } \\
\hline Small & \multicolumn{2}{|c|}{ Reference } & $<0.0001$ & \multicolumn{2}{|c|}{ Reference } & $<0.0001$ \\
\hline Medium & 1.16 & $1.11-1.21$ & & 0.86 & $0.82-0.90$ & \\
\hline Large & 1.25 & $1.20-1.30$ & & 0.80 & $0.77-0.78$ & \\
\hline \multicolumn{7}{|l|}{ Hospital location \& teaching status } \\
\hline Rural & \multicolumn{2}{|c|}{ Reference } & $<0.0001$ & \multicolumn{2}{|c|}{ Reference } & $<0.0001$ \\
\hline Urban non-teaching & 1.16 & $1.11-1.20$ & & 0.86 & $0.82-0.90$ & \\
\hline Urban teaching & 1.34 & $1.28-1.41$ & & 0.74 & $0.71-0.78$ & \\
\hline \multicolumn{7}{|l|}{ Hospital region } \\
\hline Northeast & \multicolumn{2}{|c|}{ Reference } & $<0.0001$ & \multicolumn{2}{|c|}{ Reference } & $<0.0001$ \\
\hline Midwest & 1.16 & $1.11-1.20$ & & 0.87 & $0.83-0.90$ & \\
\hline South & 1.12 & $1.09-1.16$ & & 0.89 & $0.86-0.92$ & \\
\hline West & 1.02 & $0.98-1.06$ & & 0.98 & 0.94-1.02 & \\
\hline \multicolumn{7}{|l|}{ Concurrent conditions and risk factors } \\
\hline Diabetes & 0.71 & $0.69-0.74$ & $<0.0001$ & 1.40 & $1.36-1.45$ & $<0.0001$ \\
\hline Hypertension & 0.32 & $0.32-0.33$ & $<0.0001$ & 3.09 & $3.01-3.17$ & $<0.0001$ \\
\hline Obesity & 2.26 & 2.18-2.34 & $<0.0001$ & 0.44 & $0.43-0.46$ & $<0.0001$ \\
\hline Hypercholesterolemia/triglyceridemia & 0.80 & $0.77-0.84$ & $<0.0001$ & 1.24 & $1.19-1.30$ & $<0.0001$ \\
\hline Drug abuse & 2.56 & $2.44-2.68$ & $<0.0001$ & 0.39 & $0.37-0.41$ & $<0.0001$ \\
\hline Alcohol abuse & 0.75 & $0.71-0.78$ & $<0.0001$ & 1.34 & $1.28-1.41$ & $<0.0001$ \\
\hline Past history of smokin & 1.20 & $1.17-1.24$ & $<0.0001$ & 0.83 & $0.81-0.86$ & $<0.00$ \\
\hline
\end{tabular}




\begin{tabular}{|c|c|c|c|c|c|c|}
\hline Current tobacco dependence & 0.64 & $0.61-0.67$ & $<0.0001$ & 1.56 & $1.48-1.65$ & $<0.0001$ \\
\hline Ischemic heart disease & 0.46 & $0.44-0.47$ & $<0.0001$ & 2.20 & $2.11-2.28$ & $<0.0001$ \\
\hline Infective endocarditis & 2.08 & $1.67-2.58$ & $<0.0001$ & 0.48 & $0.39-0.60$ & $<0.0001$ \\
\hline Atrial fibrillation & 0.24 & $0.23-0.26$ & $<0.0001$ & 4.18 & $3.93-4.45$ & $<0.0001$ \\
\hline Cardiomyopathy & 2.11 & $1.99-2.24$ & $<0.0001$ & 0.47 & $0.45-0.50$ & $<0.0001$ \\
\hline Rheumatic fever & 4.27 & $1.76-10.36$ & 0.0014 & 0.23 & $0.10-0.57$ & 0.0014 \\
\hline Rheumatoid heart disease & 0.86 & $0.78-0.95$ & 0.0027 & 1.16 & $1.05-1.28$ & 0.0027 \\
\hline Atrial septal disease & 2.46 & $2.34-2.58$ & $<0.0001$ & 0.41 & $0.39-0.43$ & $<0.0001$ \\
\hline Ventricular septal disease & 4.99 & $3.09-8.05$ & $<0.0001$ & 0.20 & $0.12-0.32$ & $<0.0001$ \\
\hline Patent ductus arteriosus & 1.37 & $0.59-3.19$ & 0.4681 & 0.73 & $0.31-1.70$ & 0.4681 \\
\hline Urinary tract infection & 0.64 & $0.61-0.67$ & $<0.0001$ & 1.56 & $1.49-1.64$ & $<0.0001$ \\
\hline HIV infection & 4.36 & $3.62-5.26$ & $<0.0001$ & 0.23 & $0.19-0.28$ & $<0.0001$ \\
\hline Pneumonia & 0.80 & $0.73-0.87$ & $<0.0001$ & 1.26 & $1.16-1.37$ & $<0.0001$ \\
\hline Neurosyphilis & 0.62 & $0.38-1.00$ & 0.0487 & 1.62 & $1.00-2.60$ & 0.0487 \\
\hline Meningitis & 1.93 & $0.84-4.45$ & 0.1223 & 0.52 & $0.23-1.19$ & 0.1223 \\
\hline CNS lymphoma & 1.55 & $0.29-8.42$ & 0.6096 & 0.64 & $0.12-3.49$ & 0.6096 \\
\hline Brain tumors & 7.89 & $5.48-11.36$ & $<0.0001$ & 0.13 & $0.09-0.18$ & $<0.0001$ \\
\hline Epilepsy & 1.43 & $1.37-1.50$ & $<0.0001$ & 0.70 & $0.67-0.73$ & $<0.0001$ \\
\hline Hemorrhagic stroke & 0.98 & $0.89-1.08$ & 0.6444 & 1.02 & $0.93-1.13$ & 0.6444 \\
\hline Arterial-venous malformation & 1.81 & $1.43-2.28$ & $<0.0001$ & 0.55 & $0.44-0.70$ & $<0.0001$ \\
\hline History of transient ischemic attack & 0.82 & $0.78-0.87$ & $<0.0001$ & 1.21 & $1.16-1.28$ & $<0.0001$ \\
\hline Traumatic brain injury & 0.70 & $0.50-0.97$ & 0.0328 & 1.43 & $1.03-2.00$ & 0.0328 \\
\hline Chronic kidney diseases & 0.63 & $0.57-0.71$ & $<0.0001$ & 1.58 & $1.42-1.75$ & $<0.0001$ \\
\hline Acute renal failure & 0.84 & $0.79-0.89$ & $<0.0001$ & 1.19 & $1.12-1.26$ & $<0.0001$ \\
\hline End-stage renal disease & 2.19 & $1.92-2.51$ & $<0.0001$ & 0.46 & $0.40-0.52$ & $<0.0001$ \\
\hline Systemic lupus erythematous & 3.76 & $2.99-4.73$ & $<0.0001$ & 0.27 & $0.21-0.34$ & $<0.0001$ \\
\hline Scleroderma & 2.25 & $0.50-10.18$ & 0.2937 & 0.45 & $0.10-2.02$ & 0.2937 \\
\hline Systemic sclerosis & 1.18 & $0.79-1.77$ & 0.4183 & 0.85 & $0.57-1.27$ & 0.4183 \\
\hline Rheumatoid arthritis & 0.44 & $0.34-0.56$ & $<0.0001$ & 2.29 & $1.79-2.93$ & $<0.0001$ \\
\hline Polymyositis & 2.72 & $1.26-4.69$ & 0.0105 & 0.37 & $0.17-0.79$ & 0.0105 \\
\hline Dermatomyositis & 1.06 & $0.39-2.91$ & 0.9061 & 0.94 & $0.34-2.58$ & 0.9061 \\
\hline Ankylosis spondylosis & 2.42 & $1.26-4.69$ & 0.0082 & 0.41 & $0.21-0.80$ & 0.0082 \\
\hline Psoriatic arthritis & 1.10 & $0.69-1.76$ & 0.6998 & 0.91 & $0.57-1.46$ & 0.6998 \\
\hline Hypercoagulable state & 4.03 & $3.72-4.36$ & $<0.0001$ & 0.25 & $0.23-0.27$ & $<0.0001$ \\
\hline Polycythemia vera & 1.10 & $0.91-1.33$ & 0.3335 & 0.91 & $0.75-1.10$ & 0.3335 \\
\hline Giant cell arteritis & 0.24 & $0.11-0.54$ & 0.0004 & 4.11 & $1.87-9.03$ & 0.0004 \\
\hline Polyarteritis nodosa & 5.65 & $2.16-14.81$ & 0.0004 & 0.18 & $0.07-0.46$ & 0.0004 \\
\hline Thromboangiitis obliterans & 1.86 & $0.83-4.15$ & 0.1304 & 0.54 & $0.24-1.20$ & 0.1304 \\
\hline Amyloidosis & 0.11 & $0.04-0.31$ & $<0.0001$ & 9.09 & $3.25-25.39$ & $<0.0001$ \\
\hline
\end{tabular}




\section{Cureus}

\begin{tabular}{|c|c|c|c|c|c|c|}
\hline Fibromuscular dysplasia & 2.83 & $2.20-3.65$ & $<0.0001$ & 0.35 & $0.27-0.45$ & $<0.0001$ \\
\hline \multicolumn{7}{|l|}{ Comorbidities of patients } \\
\hline Deficiency anemias & 1.15 & $1.11-1.20$ & $<0.0001$ & 0.87 & $0.83-0.90$ & $<0.0001$ \\
\hline $\begin{array}{l}\text { Rheumatoid arthritis/collagen vascular } \\
\text { diseases }\end{array}$ & 0.96 & $0.77-1.19$ & 0.6861 & 1.05 & $0.84-1.30$ & 0.6861 \\
\hline Chronic blood loss anemia & 1.82 & $1.56-2.13$ & $<0.0001$ & 0.55 & $0.47-0.64$ & $<0.0001$ \\
\hline Congestive heart failure & 0.86 & $0.81-0.91$ & $<0.0001$ & 1.17 & $1.11-1.23$ & $<0.0001$ \\
\hline Chronic pulmonary disease & 0.73 & $0.70-0.76$ & $<0.0001$ & 1.37 & $1.32-1.44$ & $<0.0001$ \\
\hline Hypothyroidism & 0.55 & $0.52-0.58$ & $<0.0001$ & 1.83 & $1.73-1.93$ & $<0.0001$ \\
\hline Liver disease & 0.76 & $0.68-0.85$ & $<0.0001$ & 1.32 & $1.18-1.47$ & $<0.0001$ \\
\hline Lymphoma & 0.81 & $0.65-0.99$ & 0.0410 & 1.24 & $1.01-1.53$ & 0.0410 \\
\hline Fluid and electrolyte disorders & 0.91 & $0.88-0.95$ & $<0.0001$ & 1.09 & $1.06-1.13$ & $<0.0001$ \\
\hline Metastatic cancer & 0.38 & $0.32-0.45$ & $<0.0001$ & 2.63 & 2.23-3.12 & $<0.0001$ \\
\hline Paralysis & 1.45 & $1.36-1.53$ & $<0.0001$ & 0.69 & $0.65-0.73$ & $<0.0001$ \\
\hline Psychoses & 1.48 & $1.39-1.57$ & $<0.0001$ & 0.68 & $0.64-0.72$ & $<0.0001$ \\
\hline Peptic ulcer disease excluding bleeding & 0.43 & $0.16-1.17$ & 0.0984 & 2.35 & $0.85-6.45$ & 0.0984 \\
\hline Valvular disease & 1.08 & $1.02-1.14$ & 0.0090 & 0.93 & $0.88-0.98$ & 0.0090 \\
\hline Weight loss & 0.65 & $0.59-0.71$ & $<0.0001$ & 1.55 & $1.41-1.70$ & $<0.0001$ \\
\hline Pulmonary circulation disorders & 0.90 & $0.82-0.99$ & 0.0273 & 1.11 & $1.01-1.23$ & 0.0273 \\
\hline Coagulopathy & 1.05 & $0.97-1.13$ & 0.2226 & 0.95 & $0.88-1.03$ & 0.2226 \\
\hline Solid tumor without metastasis & 0.22 & $0.18-0.26$ & $<0.0001$ & 4.65 & $3.81-5.68$ & $<0.0001$ \\
\hline Depression & 1.14 & 1.09-1.19 & $<0.0001$ & 0.88 & $0.84-0.92$ & $<0.0001$ \\
\hline Peripheral vascular disease & 1.18 & $1.13-1.24$ & $<0.0001$ & 0.84 & $0.81-0.88$ & $<0.0001$ \\
\hline Other neurological disorders & 1.25 & $1.08-1.44$ & 0.0028 & 0.80 & $0.69-0.93$ & 0.0028 \\
\hline Deyo-Charlson Comorbidity Index (CCI) & 0.94 & $0.93-0.95$ & $<0.0001$ & 1.06 & 1.05-1.08 & $<0.0001$ \\
\hline c-Index & $0.898>>>>>3$ & & & $0.898>>>>>3$ & & \\
\hline
\end{tabular}

TABLE 4: Traditional and non-traditional risk factors of AIS among young adults in comparison to old adults

aOR: adjusted odds ratio; Cl: confidence interval; UL: upper limit, LL: lower limit; CNS: central nervous system; AIS: acute ischemic stroke

The odds of having diabetes (aOR: 1.40; $\mathrm{p}<0.0001)$, hypertension (aOR: 3.09; $\mathrm{p}<0.0001$ ),

hypercholesterolemia/triglyceridemia (aOR: 1.24; $\mathrm{p}<0.0001$ ), alcohol abuse (aOR: 1.34; $\mathrm{p}<0.0001$ ), current tobacco dependence (aOR: 1.56; $\mathrm{p}<0.0001$ ), ischemic heart disease (aOR: $2.20 ; \mathrm{p}<0.0001$ ), atrial fibrillation (aOR: 4.18; $\mathrm{p}<0.0001$ ), rheumatoid heart disease (aOR: 1.16; $\mathrm{p}=0.0027)$, urinary tract infection (aOR: 1.56 ; $\mathrm{p}<0.0001)$, pneumonia (aOR: $1.26 ; \mathrm{p}<0.0001)$, history of transient ischemic attack (aOR: $1.21 ; \mathrm{p}<0.0001)$, traumatic brain injury (aOR: 1.43; $\mathrm{p}=0.0328$ ), chronic kidney diseases (aOR: 1.58; $\mathrm{p}<0.0001$ ), acute renal failure (aOR: 1.19; $\mathrm{p}<0.0001$ ), rheumatoid arthritis (aOR: 2.29; $\mathrm{p}<0.0001)$, giant cell arteritis (aOR: 4.11; $\mathrm{p}=0.0004$ ), amyloidosis (aOR: 9.09; $\mathrm{p}<0.0001$ ), solid tumor without metastasis (aOR: $4.65 ; \mathrm{p}<0.0001)$ and with metastasis (aOR: 2.63 ; $\mathrm{p}<0.0001$ ) were significantly higher among OA patients admitted with AIS. The $\mathrm{c}$ statistic was $0.898(>0.5)$ which indicate good models.

\section{Secondary outcomes}

Table 5 includes outcomes of AIS hospitalizations, comparing YA to OA. The all-cause in-hospital mortality ( $2.73 \%$ vs. 5.33 ; $\mathrm{p}<0.0001)$, morbidity (7.15\% vs. 7.73 ; $\mathrm{p}<0.0001)$, major/extreme loss of function $(30.7 \%$ vs. $37.21 \%$; $p<0.0001)$, and major/extreme likelihood of death (13.43\% vs. $21.62 \%$; $p<0.0001)$ were lower among YA than OA AIS hospitalizations. YA AIS hospitalizations had a higher prevalence of discharge to home 


\section{Cureus}

(64.59\% vs. 36.15\%; $\mathrm{p}<0.0001)$ than OA. The trend of all-cause in-hospital mortality in YA decreased from $4.11 \%$ in 2003 to $2.19 \%$ in 2014 (pTrend $<0.0001$ ) and decreased from $7.05 \%$ in 2003 to $4.47 \%$ in 2014 (PTrend $<0.0001$ ) in OA AIS hospitalizations (Figure 3). Mean length of stay (5.6 days vs. 5.4 days; $\mathrm{p}<0.0001$ ) and total cost of hospitalization were higher $(\$ 47,365$ vs. $\$ 37,669 ; \mathrm{p}<0.0001)$ in YA AIS hospitalizations than OA AIS hospitalizations (Table 5).

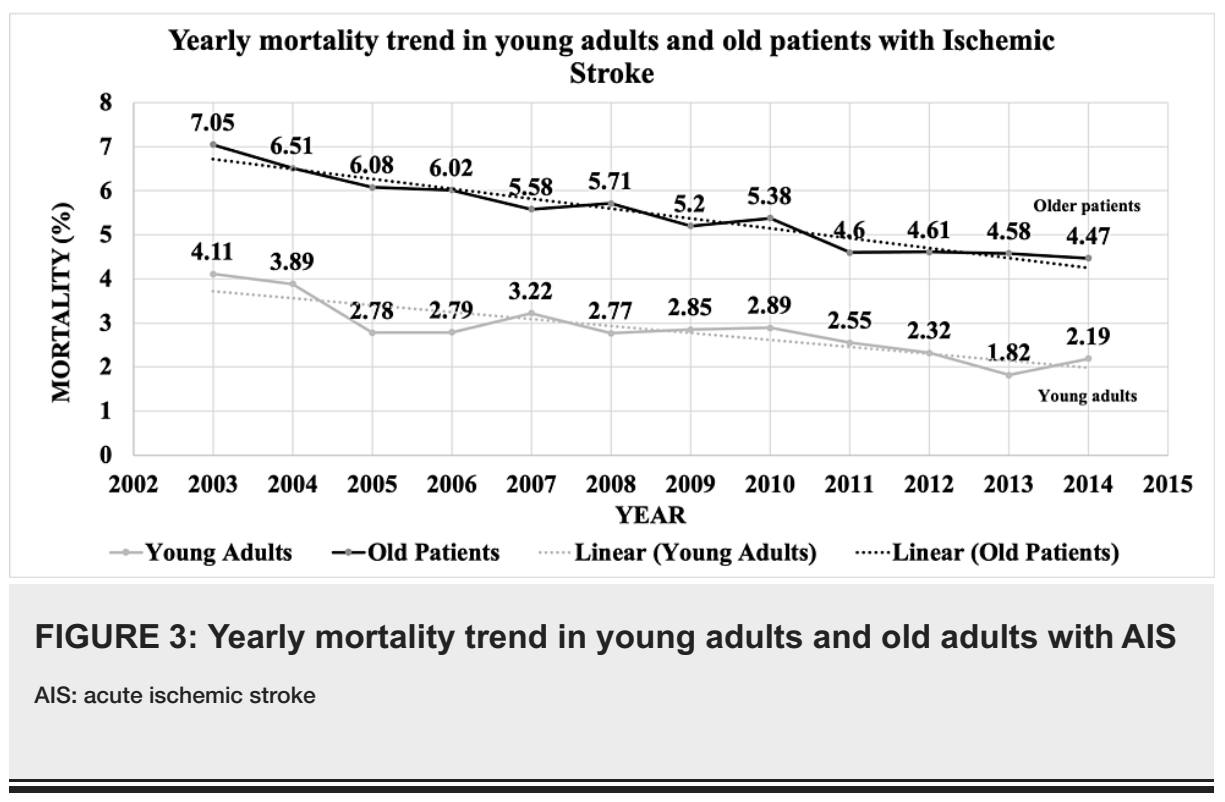




\section{Cureus}

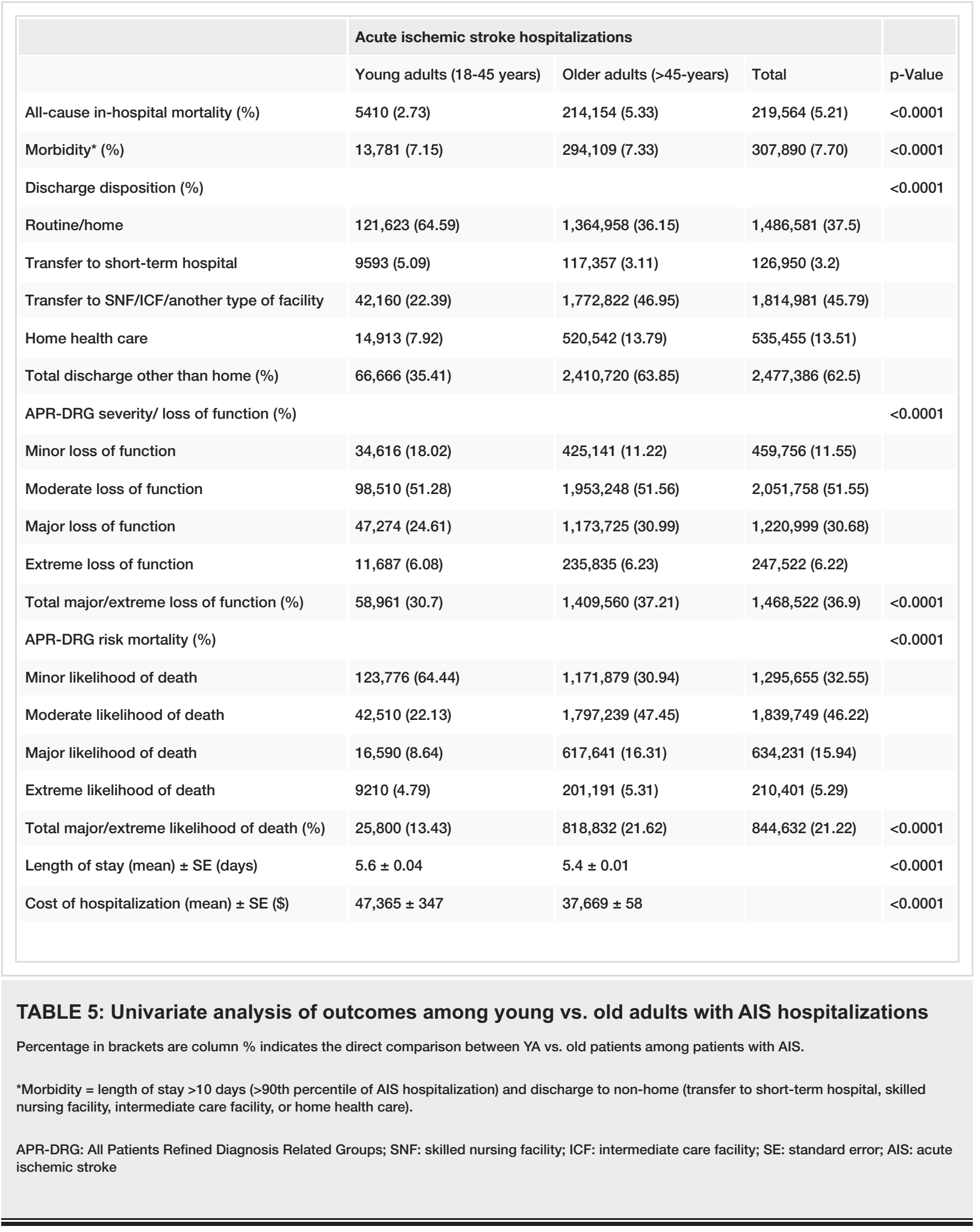

\section{Regression model derivation for the outcomes of YA}

Table 6 includes multivariable regression analysis to predict outcomes of AIS among YA and OA population. All-cause in-hospital mortality (aOR: 0.56; 95\%CI: 0.52-0.60), morbidity (aOR: 0.87; 95\%CI: 0.83-0.91), discharge disposition to non-home (aOR: 0.60; 95\%CI: 0.58-0.61), and major/extreme likelihood of death (aOR: 0.83 ; 95\%CI: 0.81-0.86) were lower among YA than OA admitted with AIS with the c-statistic of 0.672 , $0.690,0.722$, and 0.713 , respectively $(>0.5)$ which indicate good fit. 


\section{Cureus}

\begin{tabular}{|c|c|c|c|c|}
\hline \multirow{2}{*}{ Odds ratio } & \multicolumn{2}{|c|}{$95 \%$ confidence interval } & \multirow{2}{*}{ p-Value } & \multirow[t]{2}{*}{ c-Index } \\
\hline & Lower limit & Upper limit & & \\
\hline \multicolumn{5}{|c|}{ All-cause in-hospital mortality in young adults (reference: older adults) } \\
\hline 0.56 & 0.52 & 0.60 & $<0.0001$ & 0.672 \\
\hline \multicolumn{5}{|c|}{ Morbidity in young aduits (reference: older aduits)" } \\
\hline 0.87 & 0.83 & 0.91 & $<0.0001$ & 0.690 \\
\hline \multicolumn{5}{|c|}{ Discharge disposition to non-home in young adults (reference: older adults) } \\
\hline 0.60 & 0.58 & 0.61 & $<0.0001$ & 0.722 \\
\hline \multicolumn{5}{|c|}{ APR-DRG major/extreme loss of function in young adults (reference: older adults) } \\
\hline 1.02 & 0.998 & 1.05 & 0.0672 & 0.730 \\
\hline \multicolumn{5}{|c|}{ APR-DRG major/extreme risk of death in young adults (reference: older adults) } \\
\hline 0.83 & 0.81 & 0.86 & $<0.0001$ & 0.713 \\
\hline
\end{tabular}

\section{TABLE 6: Multivariable analysis of outcomes among young vs. old adults with AIS hospitalizations}

All models are adjusted for demographics (age, gender, race), patient-level hospitalization variables (admission day, primary payer, admission type, median household income category), hospital-level variables (hospital region, teaching versus nonteaching hospital, hospital bed size), comorbidities and risk factors like hypertension, diabetes mellitus, hypercholesterolemia, obesity, current smoking status, ex-smoker, drug abuse, alcohol abuse, and Charlson Comorbidity Index.

*Morbidity = length of stay $>10$ days (>90th percentile of AIS hospitalization) and discharge to non-home (transfer to short term hospital, skilled nursing facility, intermediate care facility, or home health care)

APR-DRG: All Patients Refined Diagnosis Related Groups; AIS: acute ischemic stroke

\section{Discussion}

We performed a population-based retrospective analysis of the nationally-representative NIS database to identify adult AIS hospitalizations and risk factors. Stroke in YA has been observed to be uncommon, and cerebrovascular disease reaches peak incidence later in life [19]. This observation has been confirmed in our study as we identified only $4.7 \%$ YA AIS hospitalizations, while $95.3 \%$ of hospitalizations were in patients who were 45 years or older. Thus, stroke is not a common health condition among YA. However, for those YA who do suffer a stroke, it is a considerable cause of morbidity and has an impact on the loss of work productivity in these patients [9]. Despite the small number of YA who suffer from stroke, we found an increasing prevalence among YA with AIS. From 2003 to 2014, hospitalizations for AIS in young adults increased from $4.36 \%$ to $4.70 \%$. This stands in contrast to previous reports of stable rates of stroke incidence and decreasing rates of stroke hospitalization among adults [9]. A possible reason for this seemingly increasing incidence could be due to rising rates of stroke risk factors, including obesity, hypertension, diabetes, tobacco, and alcohol use [9].

Many risk factors among YA are traditional and modifiable, so screening and treatment are possible. These include obesity, drug abuse, history of smoking, infective endocarditis, cardiomyopathy, rheumatic fever, atrial septal disease, ventricular septal disease, HIV infection, and epilepsy. Some of the non-traditional risk factors like arterial-venous malformation, brain tumors, end-stage renal disease, systemic lupus erythematosus, polymyositis, ankylosis spondylosis, hypercoagulable state, polyarteritis nodosa, and fibromuscular dysplasia are significantly associated with YA with AIS.

Notably, in our study, all-cause in-hospital mortality was lower among YA. The prevalence rate of inhospital mortality decreased from 2003 to 2014 (YA: $4.11 \%$ to $2.19 \%$ and OA: $7.05 \%$ to $4.47 \%$ ), similar to Lee et al. (1998: 7.0\% to 2007: 5.4\%; $\mathrm{p}<0.0001$ ) [20]. A possible explanation for this could be more effective treatment guidelines and strategies when presenting to the hospital. Young people may still be participating in high-risk factors that can lead to an increase in AIS hospitalizations, as shown in our study; however, treatment may have improved, with a concomitant decrease in mortality. Our study also indicated that YA with AIS hospitalizations had a lower chance of morbidity, discharge to short/long-term care, and the likelihood of death. YA and OA AIS hospitalizations had a similar mean LoS. However, the cost of hospitalizations was higher in YA ( $\$ 47365$ vs. $\$ 37669$, $p<0.0001)$. Stroke is thus an important cause of 
morbidity in young patients, and although having a small prevalence in the population, it affects hospitalization costs and dramatic impacts on quality of life in survivors. YA are also associated with higher long-term cumulative mortality due to stroke compared to the general population [21]. Stroke causes numerous physical and cognitive problems, long-term consequences, and work-related productivity losses especially in younger populations [21].

A major strength of this study was the findings that were nationally representative for the United States. However, there are limitations to this study. AIS was analyzed as a whole, rather than by identifying AIS patients according to subtype or by comparing other types of stroke. Perhaps, YA with AIS hospitalizations were due to a certain cause or presented as a subtype of AIS; however, this could not be elicited through this study. Additionally, being a retrospective study, we have associations between certain concurrent diagnoses and co-morbidities and AIS, yet we do not know if there is a temporal relationship between the two. We have evaluated in-hospital outcomes and do not have post-discharge records of these patients. Likewise, we are missing other details like stroke location, NIH Stroke Scale, concurrent medication use, the severity of risk factors, etc.

\section{Conclusions}

AIS has been shown to be an uncommon problem in YA with better outcomes; however, with the rising prevalence trend of AIS over the past decade in young populations, prevention and treatment strategies need to be examined. Young adults have modifiable risk factors such as obesity, drug and smoking abuse, and heart conditions that can be screened. Besides that, non-traditional risk factors suggest that more awareness and prevention strategies can be targeted to the YA population. Further studies should be done to test whether modifying these factors lowers stroke risk in the young population or to determine if awareness campaigns differ based on the age of the patient targeted.

\section{Additional Information \\ Disclosures}

Human subjects: Consent was obtained or waived by all participants in this study. Health Care Utilization Project (HCUP) issued approval N/A. The data has been taken from Nationwide Inpatient Sample, which is a deidentified database from "Health Care Utilization Project (HCUP)" sponsored by the Agency for Healthcare Research and Quality, so informed consent or IRB approval was not needed for the study. The relevant ethical oversight and HCUP Data Use Agreement (HCUP-4Q28K90CU) were obtained by Urvish Patel for the data used in this study. Animal subjects: All authors have confirmed that this study did not involve animal subjects or tissue. Conflicts of interest: In compliance with the ICMJE uniform disclosure form, all authors declare the following: Payment/services info: All authors have declared that no financial support was received from any organization for the submitted work. Financial relationships: All authors have declared that they have no financial relationships at present or within the previous three years with any organizations that might have an interest in the submitted work. Other relationships: All authors have declared that there are no other relationships or activities that could appear to have influenced the submitted work.

\section{References}

1. Kochanek KD, Murphy SL, Xu J, Arias E: Mortality in the United States, 2013. NCHS Data Brief. 2014, 1-8.

2. Mozaffarian D, Benjamin EJ, Go AS, et al.: Heart disease and stroke statistics-2016 update: a report from the American Heart Association. Circulation. 2016, 133:38-360. 10.1161/CIR.0000000000000350

3. Hall MJ, Levant S, DeFrances CJ: Hospitalization for stroke in U.S. hospitals, 1989-2009. NCHS Data Brief. 2012, 1-8.

4. Putaala J, Metso AJ, Metso TM, et al.: Analysis of 1008 consecutive patients aged 15 to 49 with first-ever ischemic stroke: the Helsinki young stroke registry. Stroke. 2009, 40:1195-1203. 10.1161/STROKEAHA.108.529883

5. Adams HP Jr, Kappelle LJ, Biller J, Gordon DL, Love BB, Gomez F, Heffner M: Ischemic stroke in young adults. Experience in 329 patients enrolled in the Iowa Registry of stroke in young adults. Arch Neurol. 1995, 52:491-495. 10.1001/archneur.1995.00540290081021

6. Kittner SJ, Stern BJ, Wozniak M, et al.: Cerebral infarction in young adults: the Baltimore-Washington Cooperative young stroke study. Neurology. 1998, 50:890-894. 10.1212/wnl.50.4.890

7. Jacobs BS, Boden-Albala B, Lin IF, Sacco RL: Stroke in the young in the northern Manhattan stroke study . Stroke. 2002, 33:2789-2793. 10.1161/01.str.0000038988.64376.3a

8. Naess H, Nyland HI, Thomassen L, Aarseth J, Nyland G, Myhr KM: Incidence and short-term outcome of cerebral infarction in young adults in western Norway. Stroke. 2002, 33:2105-2108. 10.1161/01.str.0000023888.43488.10

9. George MG, Tong X, Kuklina EV, Labarthe DR: Trends in stroke hospitalizations and associated risk factors among children and young adults, 1995-2008. Ann Neurol. 2011, 70:713-721. 10.1002/ana.22539

10. George MG, Tong X, Bowman BA: Prevalence of cardiovascular risk factors and strokes in younger adults . JAMA Neurol. 2017, 74:695-703. 10.1001/jamaneurol.2017.0020

11. Smajlović D: Strokes in young adults: epidemiology and prevention . Vasc Health Risk Manag. 2015, 11:157164. 10.2147/VHRM.S53203

12. Poisson SN, Glidden D, Johnston SC, Fullerton HJ: Deaths from stroke in US young adults, 1989-2009. Neurology. 2014, 83:2110-2115. 10.1212/WNL.0000000000001042 


\section{Cureus}

13. Fromm A, Waje-Andreassen U, Thomassen L, Naess H: Comparison between ischemic stroke patients $<50$ years and $\geqslant 50$ years admitted to a single centre: the Bergen stroke study. Stroke Res Treat. 2011, 2011:183256. 10.4061/2011/183256

14. Edwards JD, Kapral MK, Fang J, Swartz RH: Long-term morbidity and mortality in patients without early complications after stroke or transient ischemic attack. CMAJ. 2017, 189:954-961. 10.1503/cmaj.161142

15. Birman-Deych E, Waterman AD, Yan Y, Nilasena DS, Radford MJ, Gage BF: Accuracy of ICD-9-CM codes for identifying cardiovascular and stroke risk factors. Med Care. 2005, 43:480-485.

10.1097/01.mlr.0000160417.39497.a9

16. Thirumala PD, Nguyen FD, Mehta A, et al.: Perioperative stroke, in-hospital mortality, and postoperative morbidity following transcatheter aortic valve implantation: a nationwide study. J Clin Neurol. 2017, 13:351358. 10.3988/jcn.2017.13.4.351

17. McCormick PJ, Lin HM, Deiner SG, Levin MA: Validation of the All Patient Refined Diagnosis Related Group (APR-DRG) risk of mortality and severity of illness modifiers as a measure of perioperative risk. J Med Syst. 2018, 42:81. 10.1007/s10916-018-0936-3

18. Baram D, Daroowalla F, Garcia R, et al.: Use of the All Patient Refined-Diagnosis Related Group (APR-DRG) risk of mortality score as a severity adjustor in the medical ICU. Clin Med Circ Respirat Pulm Med. 2008, 2:19-25. 10.4137/ccrpm.s544

19. Burns RJ, Blumbergs PC, Sage MR: Brain infarction in young men. Clin Exp Neurol. 1979, 16:69-79.

20. Lee LK, Bateman BT, Wang S, Schumacher HC, Pile-Spellman J, Saposnik G: Trends in the hospitalization of ischemic stroke in the United States, 1998-2007. Int J Stroke. 2012, 7:195-201. 10.1111/j.17474949.2011.00700.x

21. Maaijwee NA, Rutten-Jacobs LC, Schaapsmeerders P, van Dijk EJ, de Leeuw FE: Ischaemic stroke in young adults: risk factors and long-term consequences. Nat Rev Neurol. 2014, 10:315-325.

10.1038/nrneurol.2014.72 